Valentin B. KATKALOV, Senior Researcher, Military (Research) Institute laboratory, Mozhaisky Military Space Academy,

St. Petersburg, Russia, vkatkalov@mail.ru

ABSTRACT I The article discusses the status and development prospects of space satellite services using the example of MEV and MEP spacecraft. The principle of operation and purpose of service spacecraft are considered. The problems arising during the creation of satellite services are estimated. The main directions of the provision and application of satellite services for solving targets are shown.

Keywords: Service spacecraft (SKA), extension of the spacecraft (SC) resource, refueling of spacecraft, changing, maintaining the orbit and orientation of satellites, automated repairs on board, technical inspection

\title{
SPACE SERVIGES AND OPERATIONS. STATE AND PROSPECTS
}




\section{КОСМИЧЕСКИЕ УСЛУГИИОПЕРАЦИИ: COCTOAHИЕ И ПЕРСПЕКТИВЫ}

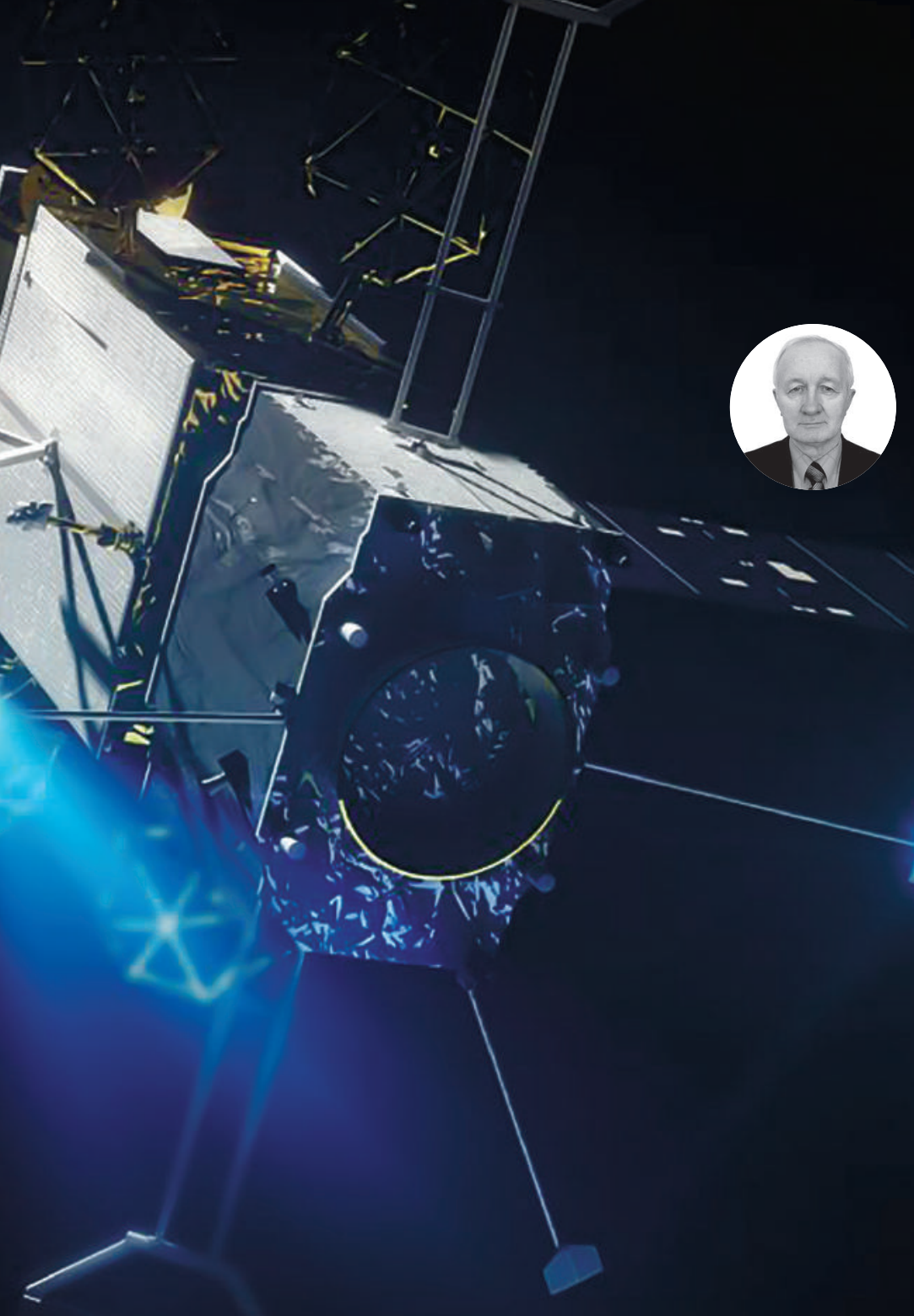

Валентин Борисович КАТЬКАЛОВ,

старший научный сотрудник лаборатории Военного

института (научно-исследовательского) Военно-космической академии имени А.Ф. Моккайского, Санкт-Петербург, Россия,

vkatkalov@mail.ru

АННООТАЦИЯ I В статье проанализированы состояние и перспективыразвития космических спутниковых услуг на примере космических аппарат̈ов MEV и MEP, рассмотрены принцип действия и назначение сервисных космических аппаратов. Оценены проблемы, возникающие при создании спутниковых услуг. Показаны основные направления оказания спутниковых услуг для решения целевых задач.

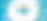

(4) очевые слова: сервисный космический аппарат (CKA), продление ресурса космических аппаратов (KA), заправка космических аппаратов топливом, изменение, поддержание орбиты и ориентации спутников, автоматизированные ремонты на борту, техническая инспекция 


\section{ВВЕДЕНИЕ}

За последние десятилетия космические аппараты на орбите, как старые, так и новые, начали требовать внимания не только с позиции текущих операций, но и для продолжения работы в перспективе. И если малые космические аппараты (MKA) в силу их относительной дешевизны в случае отказа можно заменить на орбите другим аналогичным КА, то дорогие и большие КА и системы в приемлемые сроки заменить невозможно. Многие успешно функционирующие в настоящее время КА на различных орбитах в ближайшем будущем окажутся без топлива, могут оказаться без солнечных батарей, а также без каких-либо функциональных элементов. На Земле подобная проблема решилась бы, по космическим меркам, практически мгновенно. Но КА находятся на орбите. Отсюда возникает необходимость переноса эксплуатационного обслуживания (услуг) в космос [1].

\section{АНАЛИЗ СОВРЕМЕННОГО СОСТОЯНИЯ КОСМИЧЕСКИХ АППАРАТОВ И СИСТЕМ, НАХОДЯЩИХСЯ В ЭКСПЛУАТАЦИИ НА ЦЕЛЕВЫХ ОРБИТАХ}

Космические аппараты и системы, созданные за последние десятилетия, в количественном составе выросли в геометрической прогрессии. Для того чтобы КА работали долго, требуется энергия, топливо, а возможно и рациональная модернизация. Но все это можно обеспечить только с помощью специальных сервисных космических аппаратов (СKA), посредством которых можно проводить определенные операции в космосе с целью продления ресурса уникального КА и соответственно - срока его существования (рис. 1), безусловно, при соответствующем расчете и анализе экономического эффекта. Прежде всего это касается дорогостоящих космических аппаратов связи и дистанционного зондирования Земли (ДЗ3).
Анализируя современное состояние космических аппаратов и систем, находящихся в эксплуатации на целевых орбитах, можно прийти к выводу, что по принципу сервисного обслуживания можно работать и со старыми КА, не имеющими унифицированных узлов стыковки с CKA. Подтверждением тому является состоявшийся эксперимент по восстановлению работы спутника связи Intelsat 901. Космический аппарат MEV-1 25 февраля 2020 года впервые состыковался с указанным спутником связи, находящимся до этого на орбите захоронения, и с помощью ионных двигателей перевел его в согласованную рабочую точку стояния на геостационарной орбите.

\section{По принципу сервисного обслуживания можно работать и со старыми космическими аппарата- ми, находящимися в эксплуатации на целевых орбитах и не имеющими унифицированных узлов стыковки с сервисным аппаратом.}

Рис. 1. Демонстрация оказания технической помощи КА

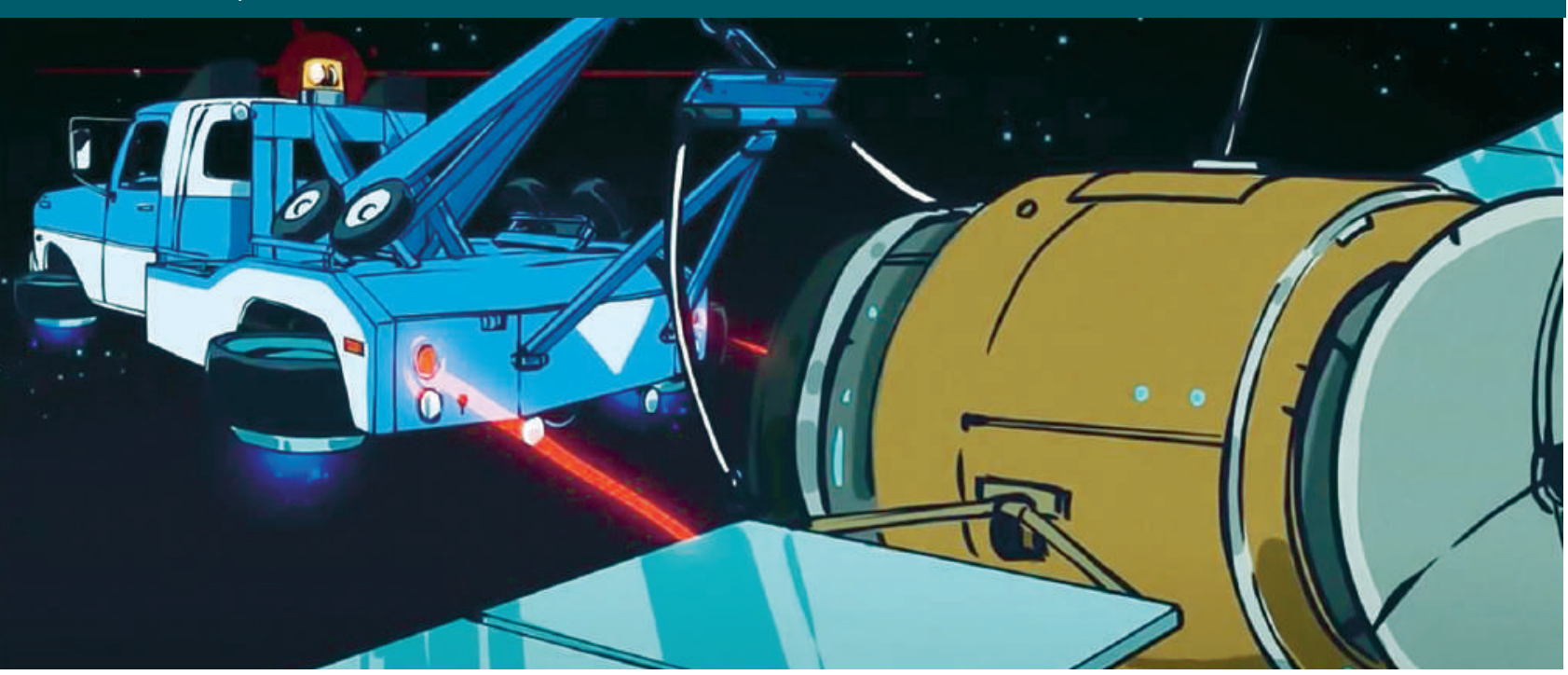




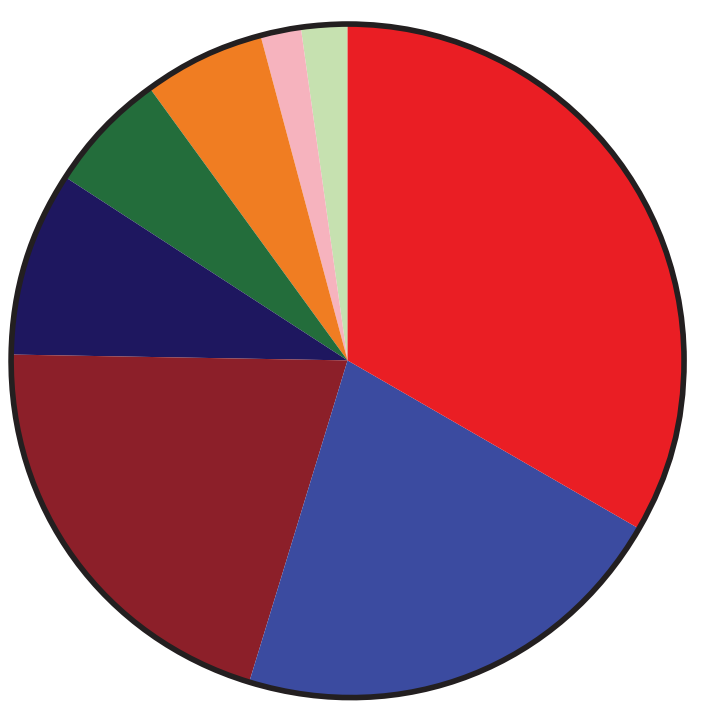

Современное состояние космических аппаратов и систем, находящихся в эксплуатации, которые могут принимать сервисное обслуживание, определяется заложенными характеристиками. Привести перечень работающих и условно работающих КА в настоящее время можно, но это потребует дополнительной публикации. Укажем, что только в 2019 году на орбиту было выведено 433 космических аппарата. Еще 141 спутник был запущен с борта МКС или отдельно от других КА. В итоге за год было запущено $574 \mathrm{KA}$, что на 28\% больше, чем в 2018 году. Для сравнения в 2017 году запустили 444 спутника.

В общей сложности в 2019 году было осуществлено 102 орбитальных запуска.

По состоянию на декабрь 2019 года орбитальная спутниковая группировка России насчитывала более 150 космических аппаратов, почти две трети которых являлись спутниками военного назначения $[2,3]$. Более подробно состав орбитальной спутниковой группировки изложен в [4]. В этой связи можно уточнить, что, судя по мировым статистическим данным, около 20 спутников, находящихся на орбитах, ежегодно после выработки топлива выходят «на пенсию».

Около 20 космических аппаратов, находящихся на орбитах, ежегодно после выработки топлива выходят «на пенсию». Срок их действия можно и нужно продлевать с помощью сервисного обслуживания.

\begin{tabular}{|c|c|c|c|}
\hline Страна & $\begin{array}{l}\text { Количество } \\
\text { запусков }\end{array}$ & Успешных & Неудачных \\
\hline$\star$ & 34 & 32 & 2 \\
\hline & 22 & 22 & \\
\hline & 21 & 21 & \\
\hline & 9 & 8 & 1 \\
\hline & 6 & 6 & \\
\hline (7) & 6 & 6 & \\
\hline & 2 & 2 & \\
\hline () & 2 & & 2 \\
\hline Всего & 102 & 97 & 5 \\
\hline
\end{tabular}

Таким образом, исходя из изложенного очень краткого анализа, можно понять, что на орбитах функционирует значительное количество $\mathrm{KA}$, в большинстве своем - несерийная техника, для которой может понадобиться сервисное обслуживание. Интересный материал на эту тему представлен в [5].

\section{ПРЕДПОСЫЛКИ ДЛЯ СОЗДАНИЯ СИСТЕМЫ КОСМИЧЕСКИХ УСЛУГ (ОПЕРАЦИЙ) ДЛЯ ДЕЙСТВУЮЩИХ И ТРЕБУЮЩИХ РЕМОНТА КОСМИЧЕСКИХ АППАРАТОВ И СИСТЕМ}

Несмотря на то, что под каждую задачу создаются новые персональные космические аппараты, на орбитах продолжают функционировать и ранее запущенные космические аппараты. Почему бы, с целью продления ресурса, не организовать обслуживание космической техники на орбите? Это может быть инспекция, модернизация, ремонты, заправка. Пока такие операции целесообразны для отдельных миссий или аппаратов [6, 7].

Достижимые проекты предполагают обслуживание спутников, которые изготавливались без учета фактора возможного обслуживания. Кроме заправок предполагается отработать операции по доставке автономных дополнительных полезных нагрузок. Эти и другие операции, которые могут быть применимы к выше указанной группировке, будут рассмотрены в данной статье на примере KA MEV-1. 


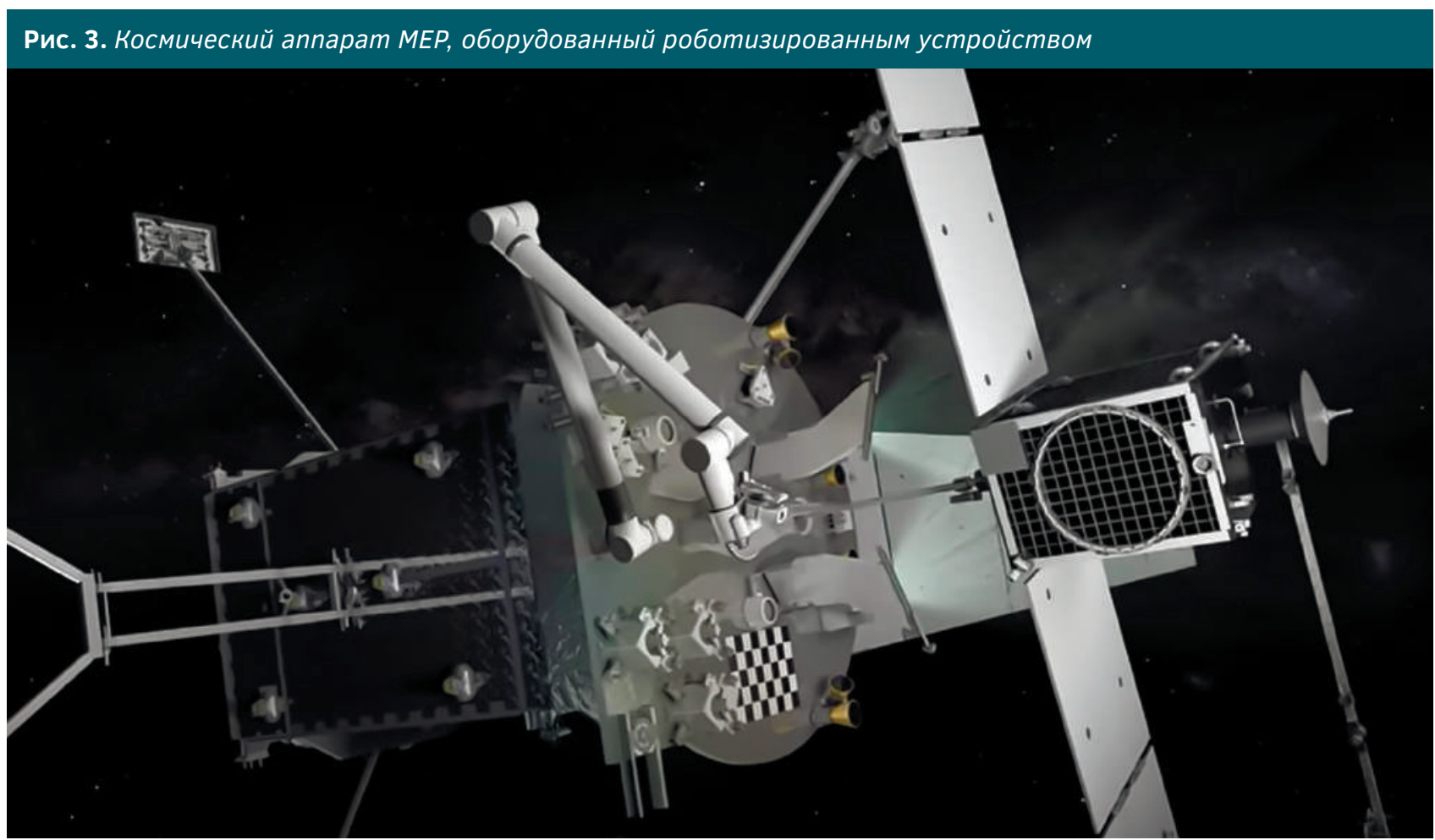

\section{ТЕХНОЛОГИЯ ПРОВЕДЕНИЯ ОПЕРАЦИИ ОБСЛУЖИВАНИЯ НА ПРИМЕРЕ КА MEV-1}

Тема космического обслуживания, несмотря на современное звучание, прослеживается уже в опыте прошлых лет. Так, 9 марта 2007 года в космос были запущены два спутника: NEXTSat, выполнявший роль цели, и ASTRO - аппарат, который осуществил с ним стыковку 29 июня того же года. В ходе испытательного полета была осуществлена стыковка, передача топлива (гидразина) и батарей [8].

На данный момент существует два проекта коммерческого обслуживания спутников - SIS и $\mathrm{MEV}$, которые существенно различаются:

1. Space Infrastructure Servicing (SIS), разрабатываемый канадской фирмой MacDonald, предусматривает кольцевой захват вокруг маршевого двигателя спутника и последующую заправку спутника;

2. Mission Extension Vehicle (MEV) от американской Northrop Grumman Corporation предусматривает установку захвата внутрь сопла (сходного с "жалом», использовавшимся на шаттлах) и не предусматривает заправки спутника-цели после стыковки спутник обслуживания будет использовать собственную систему ориентирования и свои двигатели для поддержания положения связки спутников. В этом проекте, пер- вым в отрасли, спутник MEV-1, продлевающий срок службы других спутников, завершил первую стыковку с клиентским спутником Intelsat IS-901.

Space Logistics, дочерняя компания Northrop Grumman Corporation (США), видит дальнейшую перспективу миссии и выходит далеко за рамки $\mathrm{MEV}$, планируя создать парк коммерческих KA для обслуживания функционирующих или заканчивающих свою работу КА. Этот парк будет способен удовлетворить практически любые потребности в их обслуживании, в том числе с помощью робототехники.

Первый сервисный спутник Mission Extension Vehicle $(\mathrm{MEV})^{\mathrm{TM}}$ может стыковаться только с действующими спутниками клиентов, обеспечивая маневрирование и контроль ориентации, необходимые для продления их существования. В настоящее время Space Logistics предлагает следующее поколение - систему Mission Extension Pod (MEP) ${ }^{\mathrm{TM}}$, которая является меньшей по габаритам и менее дорогостоящей системой продления функционирования КА (клиента) и которая выполняет только функцию управления его орбитой. Но в то же время МЕР оборудуется роботизированным обслуживающим транспортным средством, называемым Mission Robotic Vehicle $(M R V)^{\mathrm{TM}}$, которое может выполнять все функции MEV, добавляя новые роботизированные возможности для дополнительных услуг (рис. 3). 


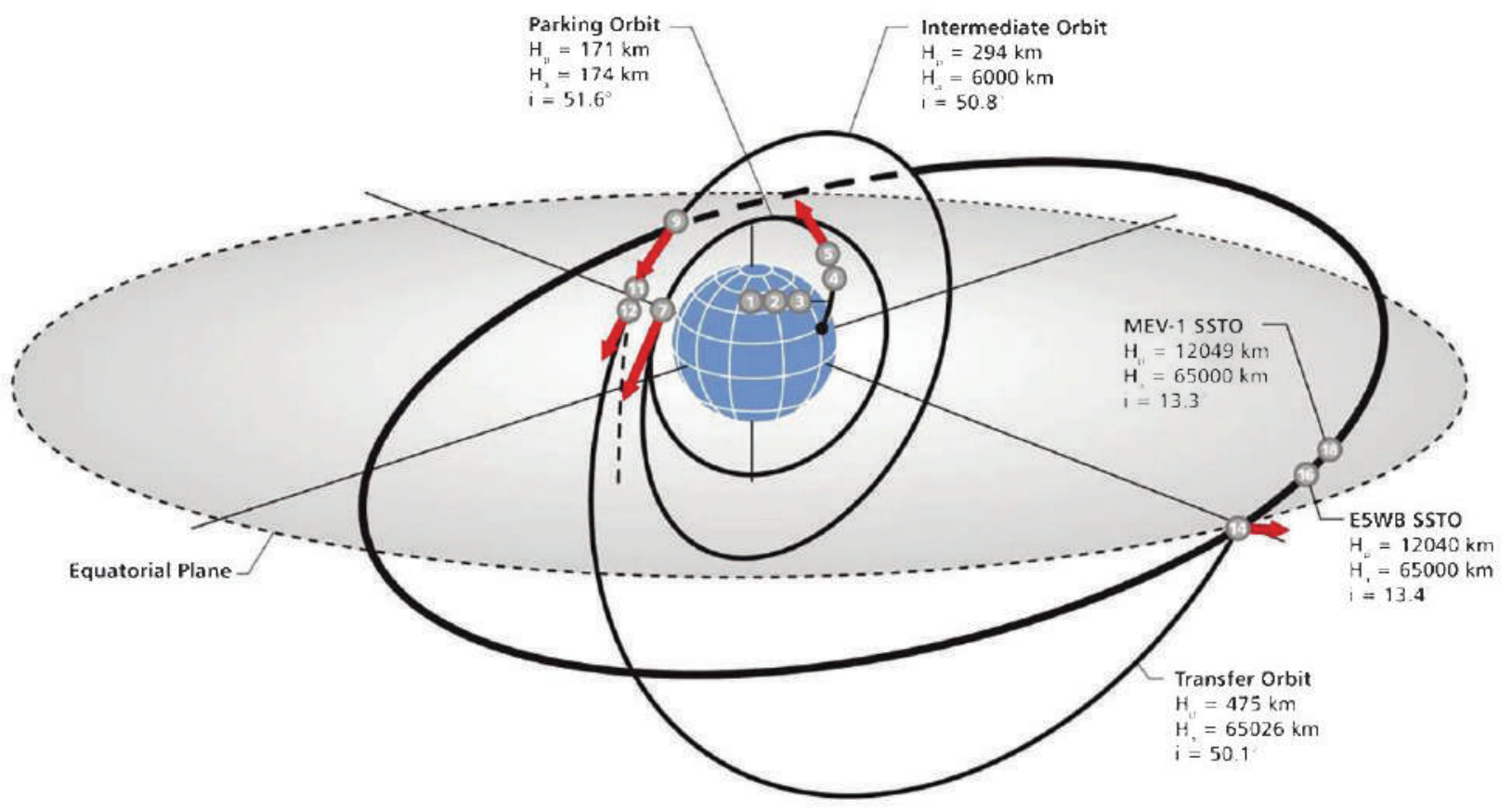

Рис. 5. Процесс стыковки MEV-1 с тыльной стороны IS-901

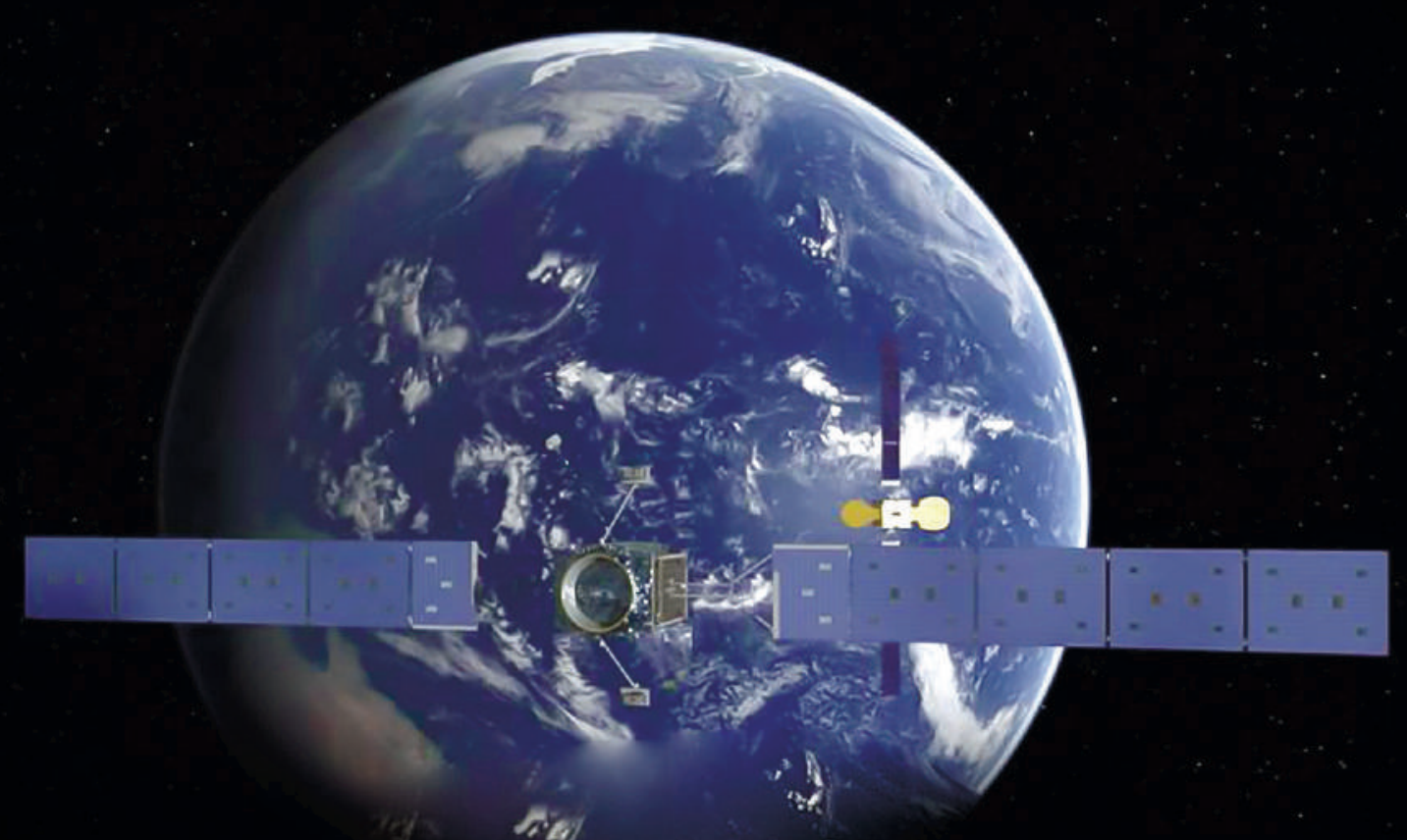

NQ? 


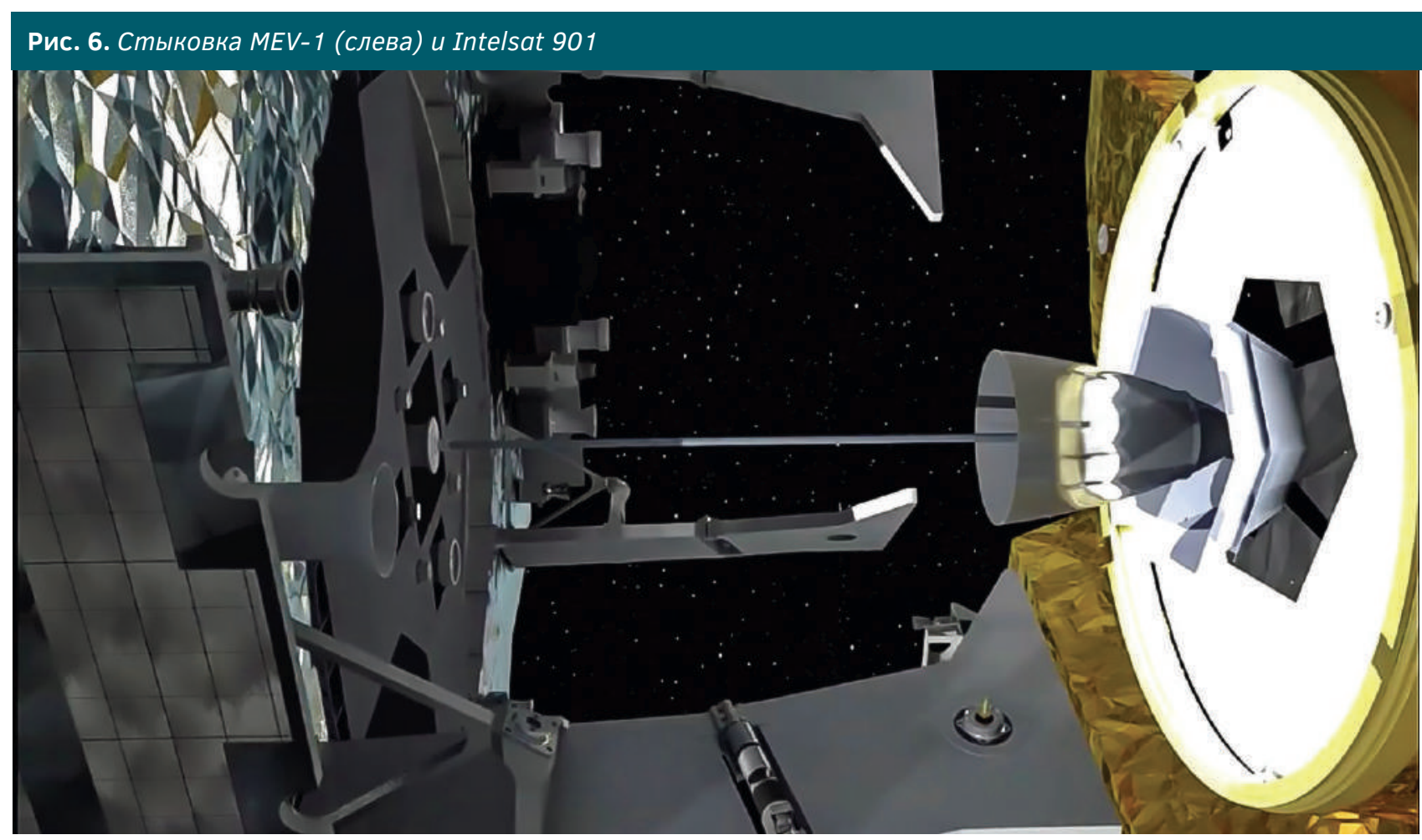

Услуги по продлению функционирования КА совместимы практически со всеми геосинхронными спутниками с минимальным перерывом в работе. Они позволяют операторам спутников связи значительно продлить срок их службы с меньшими финансовыми издержками.

Ожидается, что эти услуги будут включать:

- дополнительное увеличение емкости топлива $\mathrm{KA}$;

- осмотр и ремонт;

- замену или усовершенствование деталей и систем;

- включение вспомогательных двигателей, переход на новую систему навигации, подключение дополнительной мощности, полезных нагрузок и других функций для повышения производительности или продления срока службы спутника;

- роботизированную сборку космических структур на орбите.

Весьма подробно сервисные функции космических аппаратов MEV-1 показаны в [9 и 10].

После запуска MEV-1 отправился прямо на орбиту кладбища и поднял свою орбиту до встречи со своим клиентским спутником Intelsat 901 (IS-901) на геосинхронной экваториальной орбите (GEO). Процесс сближения и стыковки занял 3,5 месяца с момента запуска. Схему вывода и стыковку MEV можно показать наглядно с помощью нескольких рисунков и комментариев (puc. 4, 5) [11].

Метод стыковки MEV-1 чрезвычайно прост. Транспортное средство использует изделие, которым обладает примерно 80\% спутников CEO жидкостной апогейный двигатель LAE (Liquid Apogee Engine). Спутники CEO используют LAE для завершения своих орбит в начале своей работы, но никогда не используют его снова. Схема стыковки на орбите показана на рис. 6.

$\mathrm{K}$ слову, только немногие производители по всему миру строят LAE, и все они используют схожие конструкции.

Космические аппараты миссии используют запатентованный механизм для захвата и стыковки с LAE клиента. Этот механизм вставлен в конус LAE для захвата спутника-клиента - с помощью подхода, называемого "конус-захват", это тот же подход к стыковке, который использовался еще в 1960-х годах в программе Gemini [12].

Инструмент под названием «Устройство захвата маршевого двигателя", или просто "Жало» (рис. 7), вставляется в сопло маршевого двигателя спутника (так как этот двигатель размещается на линии центра масс спутника - и это самое удобное место для захвата).

После захвата механизм втягивается, и стойки MEV устанавливают прочный контакт с кольцом 


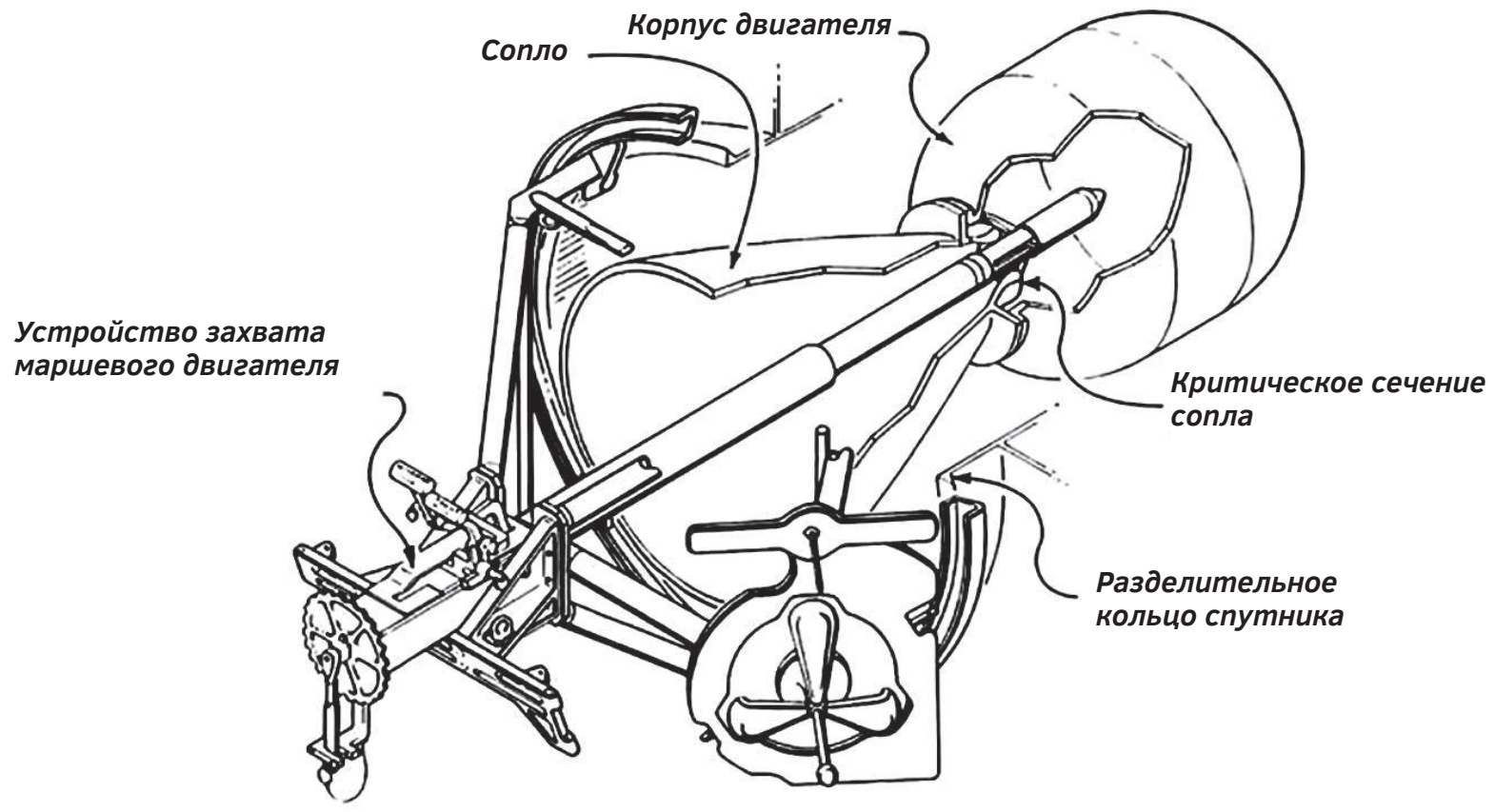

адаптера запуска транспортного средства клиента, обеспечивая безопасную стыковку.

Кольцо адаптера запуска является еще одной функцией, характерной для большинства спутников GEO. Важно отметить, что подключение транспортного средства MEV-клиент является чисто механическим, что позволяет избежать осложнений при передаче жидкости, а также электрических или информационных подключений.

После того как MEV-1 2 апреля 2020 года вернул IS-901 для обслуживания в назначенном месте в CEO, Intelsat перевел на спутник примерно 30 своих коммерческих и государственных заказчиков. Переход на обслуживание занял около шести часов. В настоящее время IS-901 работает в орбитальном слоте $332,5^{\circ} \mathrm{E}$ и обеспечивает полный сервис для клиентов Intelsat.

В конце этого контракта MEV-1 вернет IS-901 обратно на орбиту кладбища и завершит его вывод из эксплуатации.

Затем MEV-1 перейдет к предоставлению услуг по расширению миссии для новых клиентских космических аппаратов, повторив процесс еще раз и вдохнув новую жизнь в спутники, которые в ней нуждаются.

MEV-1 будет доступен в течение 15 лет для предоставления дополнительных услуг по расширению миссии для новых клиентов, вклю- чая поднятие орбиты, исправление наклона и инспекции. Intelsat уже заключил контракт c Northrop Grumman на второй MEV (MEV-2) для обслуживания спутника Intelsat 1002 в конце 2020 года $[13,14]$.

\section{ВЫВОДЫ И ЗАКЛЮЧЕНИЕ}

Исходя из вышеизложенного, с помощью СКА возможны следующие перспективы развития услуг:

1. Техническая инспекция КА, в том числе осмотр КА при помощи установленных на СКА видеокамер и предоставление информации о состоянии КА владельцу.

2. Установка на функционирующие КА дополнительной полезной нагрузки.

3. Сборка структуры космических аппаратов в определенную систему, автоматическая сборка крупных конструкций в космосе.

4. Довыведение на целевую орбиту в случае аварии разгонных блоков или спуск с орбиты космического мусора.

5. Транспортировка и подъем действующих космических аппаратов с низких круговых орбит на рабочую орбиту.

6. Корректировка положения на орбите действующего КА, у которого полностью израсходован запас рабочего тела. 
7. Монтаж и демонтаж, обслуживание функционирования КА и систем с помощью космического персонала.

В недалекой перспективе по мере развития космической техники с помощью СКА можно будет снимать с орбиты вышедшие из строя, но представляющие ценность КА, проводить чистку ГСО от отслуживших $\mathrm{KA}$, изготавливать в космосе многокилометровые каркасы космических станций, фермы антенн, базовые структуры солнечных электростанций, огромные телескопы и т.д.

Рассмотренные проблемы показывают, что космические отрасли многих государств, преследуя цель оптимизации ресурсов, приходят
В недалекой перспективе, по мере развития космической техники, с помощью сервисных космических аппаратов можно будет снимать с орбиты вышедшие из строя космические аппараты, проводить чистку геостационарной орбиты и изготавливать в космосе необходимую технику.

$\mathrm{K}$ пониманию того, что многие существующие спутники можно и нужно использовать при соответствующем продлении их ресурса без изготовления новых КА.

\section{Литература}

1. Азаренко Л.Г. Космические услуги: экономика и управление. М.: Инфра Инженерия, 2018. $252 \mathrm{c}$.

2. Более 90 из 150 российских спутников на орбите функционируют в интересах Минобороны [Электронный ресурс] // ТАСС. 05.10.2019. URL: https://tass.ru/armiya-iopk/6966714 (Дата обращения: 20.05.2020)

3. Муромова Д. Россия обновила на $80 \%$ группировку военных спутников [Электронный ресурс] // Телеканал «Звезда». 16.05.2019. URL: https://tvzvezda.ru/news/forces/ content/20195162025-g4hLr.html (Дата обращения: 20.05.2020).

4. Орбитальная спутниковая группировка России [Электронный ресурс] // WIKI 2. URL: https://wiki2.org/ru/Орбитальная спутниковая группировка России (Дата обращения: 21.05.2020)

5. Клюшников В.Ю. Современные проблемь анализа и синтеза космических систем. Тема 3. Техническое обслуживание космических аппаратов на орбите [Электронный ресурс] // Презентация MАИ, ЦНИИМаш, 2019. URL: https://ppt-online.org/678214 (Дата обращения: 12.05.2020)

6. Егоров В. Космические аппараты будущего: взгляд генконструктора [Электронный ресурс] // Ха6p.23.05.2017. URL: https://habr.com/ru/ post/404069/ (Дата обращения: 29.05.2020).

7. Клименко Н.Н., Занин К.А. Новое поколение космических аппаратов для наблюдения за морской обстановкой // Воздушно-космическая сфера. 2019. № 2. С. $72-82$.

8. Катькалов В.Б. Перспективы развития спутниковых услуг // Интеллектуальные технологии на транспорте. 2020. № 1. С. 24 - 31.

9. What is Space Logistics? [Электронный peсурс] // Northrop Grumman. URL: https://www. northropgrumman.com/space/space-logisticsservices/ (Дата обращения: 22.04.2020).

10. MEV-1 Mission Profile [Электронный ресурс] // YouTube. URL: https://youtu.be/ rgglvA5DdI (Дата обращения: 15.04.2020).

11. Успешный запуск Eutelsat-5WB и MEV-1 [Электронный ресурс] // Живой журнал. 10.10.2019. URL: https://omega-hyperon. livejournal.com/23621.html (Дата обращения 20.05.2020)

12. Прошлое и будущее обслуживания спутников [Электронный ресурс] // Хабр. 25.09.2017. URL: https://habr.com/ru/ post/406745/?fl=ru\%2Cen (Дата обращения: 27.05.2020).
13. Mission Extension Vehicle: Breathing Life Back Into In-Orbit Satellites [Электронный ресурс] // Northrop Grumman. URL: https://news. northropgrumman.com/news/features/missionextension-vehicle-breathing-life-back-into-inorbit-satellites (Дата обращения: 12.05.2020).

14. Companies demonstrate groundbreaking satellite life-extension service [Электронный peсурc] // Northrop Grumman. URL: https://news. northropgrumman.com/news/releases/northropgrumman-successfully-completes-historicfirst-docking-of-mission-extension-vehiclewith-intelsat-901-satellite (Дата обращения: 13.05.2020)

\section{References}

1. Azarenko L.G. Kosmicheskie uslugi: ekonomika i upravlenie. Moscow, Infra Inzheneriya, 2018.

$252 \mathrm{p}$.

2. Bolee 90 iz 150 rossiyskikh sputnikov na orbite funktsioniruyut v interesakh Minoborony. TASS, 2019, May 05. Available at: https://tass. ru/armiya-i-opk/6966714 (Retrieval date: 20.05.2020)

3. Muromova D. Rossiya obnovila na $80 \%$ gruppirovku voennykh sputnikov. Telekana "Zvezda", 2019, May 16. Available at: https:// tvzvezda.ru/news/forces/content/20195162025g4hLr.html (Retrieval date: 20.05.2020).

4. Orbital'naya sputnikovaya gruppirovka Rossii. WIKI 2. Available at: https://wiki2.org/ru/ Orbital'naya sputnikovaya gruppirovka Rossii (Retrieval date: 21.05.2020).

5. Klyushnikov V.Yu. Sovremennye problemy analiza i sinteza kosmicheskikh sistem. Tema 3. Tekhnicheskoe obsluzhivanie kosmicheskikh apparatov na orbite. MAI, TsNIIMash, 2019. Available at: https://ppt-online. org/678214 (Retrieval date: 12.05.2020).

6. Egorov V. Kosmicheskie apparaty budushchego: vzglyad genkonstruktora. Khabr, 2017, May 23. Available at: https://habr.com/ru/ post/404069/ (Retrieval date: 29.05.2020).

7. Klimenko N.N., Zanin K.A. Novoe pokolenie kosmicheskikh apparatov dlya nablyudeniya za morskoy obstanovkoy. Vozdushno-kosmicheskaya sfera, 2019, no. 2, pp. $72-82$

8. Kat'kalov V.B. Perspektivy razvitiya sputnikovykh uslug. Intellektual'nye tekhnologii na transporte, 2020, no. 1, pp. $24-31$.

9. What is Space Logistics? Northrop Grumman. Available at: https://www.northropgrumman. com/space/space-logistics-services/ (Retrieval date: 22.04.2020)
10. MEV-1 Mission Profile. YouTube. Available at: https://youtu.be/ rgglvA5DdI (Retrieval date: 15.04.2020) 11. Uspeshnyy zapusk Eutelsat-5WB i MEV-1. Zhivoy zhurnal, 2019, October 10. Available at: https://omega-hyperon.livejournal. com/23621.html (Retrieval date: 20.05.2020).

12. Proshloe i budushchee obsluzhivaniya sputnikov. Khabr, 2017, September 25. Available at: https://habr.com/ru/ post/406745/?fl=ru\%2Cen (Retrieval date: 27.05.2020).

13. Mission Extension Vehicle: Breathing Life Back Into In-Orbit Satellites. Northrop Grumman. Available at: https://news.northropgrumman. $\mathrm{com} /$ news/features/mission-extension-vehiclebreathing-life-back-into-in-orbit-satellites (Retrieval date: 12.05.2020).

14. Companies demonstrate groundbreaking satellite life-extension service. Northrop Grumman. Available at: https://news. northropgrumman.com/news/releases/ northrop-grumman-successfully-completeshistoric-first-docking-of-mission-extensionvehicle-with-intelsat-901-satellite (Retrieval date: 13.05.2020).

(C) Катькалов В.Б., 2020

История статьи:

Поступила в редакцию: 16.03.2020

Принята к публикации: 24.04.2020

Модератор: Плетнер К.В.

Конфликт интересов: отсутствует

Для цитирования:

Катькалов В.Б. Космические услуги и операции: состояние и перспективы // Воздушно-космическая сфера. 2020. № 2. C. $72-80$. 